\title{
Reliability Assessment of a Hexagon Socket Head Bolt Based on Moment Method
}

\author{
Xiaowei Yin $^{1}$, Wenxue Qian ${ }^{2}$ a , and Liyang Xie $^{2}$ \\ ${ }^{1}$ Department of Mechanical Engineering, Shenyang Institute of Engineering, Shenyang 110136, \\ China \\ ${ }^{2}$ School of Mechanical Engineering and Automation, Northeastern University, Shenyang 110819 , \\ China \\ aqwx99@163.com
}

Keywords: Hexagon socket head bolt, Reliability assessment, Stress - strength interference.

\begin{abstract}
Hexagon socket head bolts are widely used in many engineering fields as fasteners and are common and important parts for engineering, but for some hexagon socket head bolts it is still tedious to build the 3D models and assess their reliability. It is important for a hexagon socket head bolt to do reliability assessment, especially for some hexagon socket head bolt that under complex load conditions. In order to improve the reliability and stabilization, a hexagon socket head bolt reliability assessment method is introduced and an example is given, and the example shows that do reliability assessment is useful and effective.
\end{abstract}

\section{Introduction}

For a long period, designers have applied the method called factors of safety in a deterministic approach. But this deterministic approach is not very precise and the tendency is to use it conservatively resulting in over designed parts, high cost and sometimes ineffectiveness. So do reliability assessment is necessary. Usually a lot of hypothesis should be done, but it often causes many mistakes. Industry can progress effectively with the knowledge and implementation of reliability engineering [1-5]. Hexagon socket head bolts are wildly used in engineering, and it is a common work for an engineer to build a 3-D model of a hexagon socket head bolt with modeling software. Because there are many kind of hexagon socket head bolts, including spur hexagon socket head bolt, inner spur hexagon socket head bolt, bevel hexagon socket head bolt, helical hexagon socket head bolt etc. sometimes it is a difficult work to build a hexagon socket head bolt model quickly and accurately. It is well known that more than 80 percent of machinery failure is due to fatigue failure, and the local stress is the most important factor to decide the life of mechanical part [6-9]. It is meaningful to build a precision model of hexagon socket head bolt; an example is to do a finite element analysis of it. In this paper, an approach to assess the reliability of a spur hexagon socket head bolt is presented, and the assessment and steps will be discussed detail,For a long time the reliability of products attract more and more regard. Usually reliability is defined as the probability of a device performing its intended function for a specified period of time under the specified operating environment. This concept of reliability as a probability, typically quantified by assessing the mean time to failure (MTTF), implies that field failures are quite inevitable. In today's very competitive mechanical products market, a commitment to product reliability is necessary. Estimating produce reliability is an important and challenging job. Now the modern machine products, such as large machine tools, cars, ships etc are more and more complex and large. Also as the sophistication and complexity of systems increases, the need for subsystems and components with high reliability levels increases.

\section{Reliability Theory and Model}

The main theory about reliability design and assessment of a hexagon socket head bolt is stress strength interference theory. 
Suppose that the strength is $\delta$ and the stress is $\mathrm{s}$, of course $\mathrm{S}$ and $\mathrm{s}$ are both random variables. When the strength of a component is greater than the stress of it, the component does not fail. Suppose that the probability density function of strength is $g(\delta)$ and the distribution function is $G(\delta)$, the probability density function of stress is $\mathrm{f}(\mathrm{s})$ and the distribution function is $\mathrm{F}(\mathrm{s})$. The density function curves are shown as Fig.1.

The reliability of part is

$\mathrm{R}=\mathrm{P}(\delta>\mathrm{s})$

From Fig. 1 it can be seen when the probability density curve of strength and probability density curve of stress don not interfere and the min strength greater than the max working stress, there is

$\mathrm{R}=\mathrm{P}(\delta>\mathrm{s})=1$

The part does not fail.

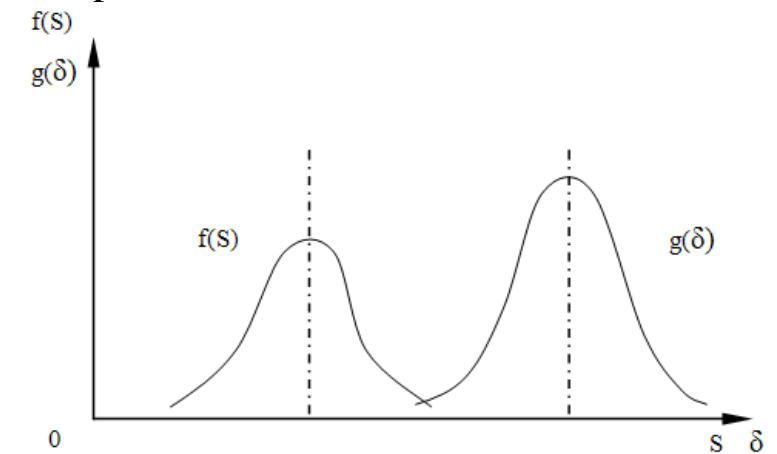

Fig.1 Distribution curve of strength and stress

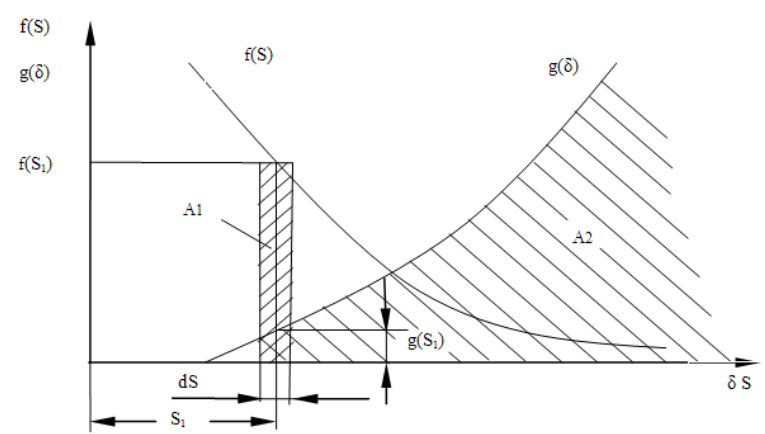

Fig.2 Interfere of strength and stress curves

When the probability density curve of strength and probability density curve of stress interfere, the part possible fail, despite the fact that the mean of stress being less than the mean of strength. Fig.2 shows the interference between probability density curve of strength and probability density curve of stress.

The probability that stress is in interval ds is:

$P\left[\left(s \frac{\mathrm{ds}}{2}\right) \leq s \leq\left(s+\frac{\mathrm{ds}}{2}\right)\right]=f(s) \mathrm{ds}$

The probability that the strength greater than the stress is:

$$
P(\delta>s)=\int_{s}^{\infty} g(\delta) \mathrm{d} \delta
$$

$\delta$ and s are independent random variables. The probability of the above two event occur at the same time is:

$$
f(s) \mathrm{d} s \times \int_{s}^{\infty} g(\delta) \mathrm{d} \delta
$$

Formula (3) is the probability that strength greater than the stress in interval ds.

Then in the entire interval, the probability that strength greater than stress is:

$$
\mathrm{R}=\int_{\infty}^{\infty} f(s) \mathrm{d} s \int_{s}^{\infty} g(\delta) \mathrm{d} \delta
$$

\section{Hexagon Socket Head Bolt Parameters and Reliability Assessment}

\subsection{D Model and Parameters of Hexagon socket head bolt.}

A hexagon socket head bolt is used in a reducer showed as Figure 3, and the parameters of it are as follows in Table 1. 
Table 1 Linear elastic analysis material parameters of wheel hub

\begin{tabular}{c|c|c|c|c}
\hline $\begin{array}{c}\text { Material } \\
\text { parameters }\end{array}$ & $\begin{array}{c}\text { Elastic module } \\
(\mathrm{MPa})\end{array}$ & $\begin{array}{c}\text { Strength limit } \\
(\mathrm{MPa})\end{array}$ & Poisson ratio & $\begin{array}{c}\text { Density } \\
(\mathrm{t} / \mathrm{mm} 3)\end{array}$ \\
\hline Value & 206000 & 800 & 0.29 & $7.8 \mathrm{E}-9$ \\
\hline
\end{tabular}

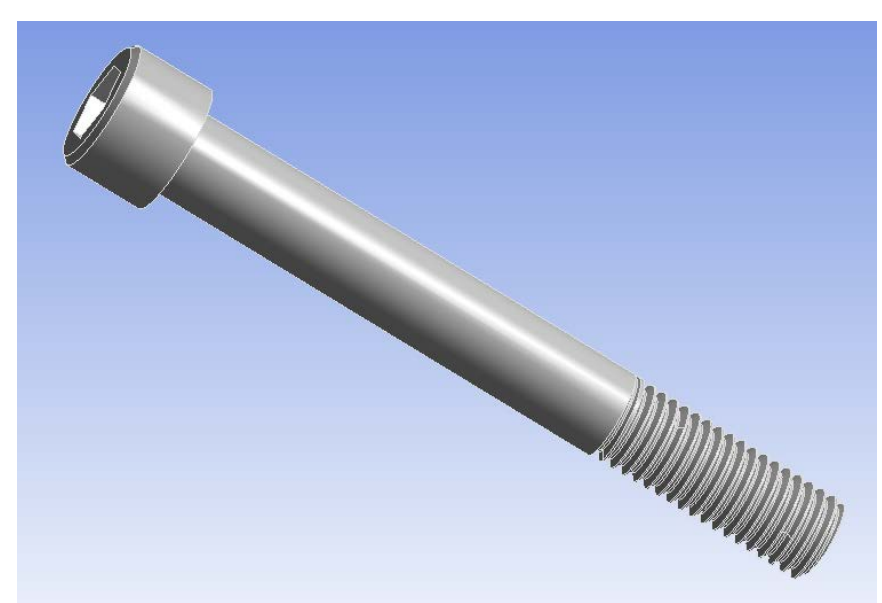

Fig.3 3D model of reducer hexagon socket head bolt

\subsection{Reliability Assessment of Hexagon socket head bolt.}

Usually the sizes of hexagon socket head bolt are not deterministic values but random variables, of course the loads the hexagon socket head bolt bearing are not deterministic values. First the loads can be obtained by calculation or test, and the strength of a tooth can also be obtained by above method, after the strength and stress are obtained, the reliability of hexagon socket head bolt can be got through model (4).

From above data the material strength of hexagon socket head bolt are $\delta\left(\mu_{\delta}, \sigma_{\delta}\right)$, and the stress are $\mathrm{s}\left(\mu_{\mathrm{s}}, \sigma_{\mathrm{s}}\right)$, then the reliability of the hexagon socket head bolt is:

Where:

$\mu_{\delta},=800$, mean of the strength of hexagon socket head bolt

$\sigma_{\delta}=40$, standard deviation of the strength of hexagon socket head bolt

$\mu_{\mathrm{s}}=600$, mean of the stress of hexagon socket head bolt

$\sigma_{\mathrm{s}}=40$, standard deviation of the stress of hexagon socket head bolt

Then the reliability of the hexagon socket head bolt is:

$$
\mathrm{R}=\mathrm{P}(\delta>\mathrm{s})
$$

Where:

$\mathrm{R}$ is hexagon socket head bolt reliability

$\delta$ is stochastic variable of strength

$\mathrm{s}$ is stochastic variable of stress

$$
R=\int_{-\infty}^{\infty} \frac{1}{\sigma_{s} \sqrt{2 \pi}} \exp \frac{-1}{2}\left(\frac{x-\mu_{s}}{\sigma_{s}}\right)^{2} \mathrm{~d} s \int_{s}^{\infty} \frac{1}{\sigma_{\delta} \sqrt{2 \pi}} \frac{-1}{2} \exp \left(\frac{y-\mu_{\delta}}{\sigma_{\delta}}\right)^{2} \mathrm{~d} \delta
$$

Because both strength variable and stress variable are normal distribution variables, $\mathrm{R}$ can be obtain through bellow formulas.

$$
\beta=\frac{\mu_{\delta}-\mu_{s}}{\sqrt{\sigma_{\delta}^{2}+\sigma_{s}^{2}}}=3.53553656
$$

Then

$$
R=\Phi(\beta)=0.99979
$$




\section{Conclusion}

Because the sizes and loads of a hexagon socket head bolt bearing are random variables, when using a deterministic method, usually it can be seen that the design result being too conservative in practical engineering. Using reliability design and assessment method, the result is much more reasonable and economy.

\section{Acknowledgments}

This work was partially supported by the National Natural Science Foundation of China (Grant No. 51335003, 51305275, 51275221, 51005044), the Fundamental Research Funds for the Central Universities (Grant No. N140301001), and the Program for Liaoning Excellent Talents in University (Grant No. LR2015044).

\section{References}

[1] Qian Wenxue, Yin Xiaowei, Xie Liyang, Complex System Reliability using Bayesian Networks, Proceedings of The 15th International Conference on Industrial Engineering and Engineering Management, A (2008) 176-179.

[2] Liyang Xie, Jinyu Zhou, Changzhong Hao, System-level load-strength interference based reliability modeling of k-out-of-n system, Reliability Engineering and System Safety, 84 (2004) 311-317.

[3] Ghosh S, Bhandari Y, Groeber M., CAD-based reconstruction of 3D polycrystalline alloy microstructures from FIB generated serial sections, CAD Computer Aided Design, 40 (2008) 293-310.

[4] Billo Richard E, Bidanda Bopaya, Peternel Jim, Parametric design of countersink cutting tool. Proceedings of the Industrial Engineering Research Conference, (1993) 370-374.

[5] Qian Wenxue, Xie Liyang, Huang Dayan, Yin Xiaowei, Boltt Parametric Design System Development Based on ProEngineer, The Second International Conference on Information and Computing Science, 1 (2009) $42-45$.

[6] Wenxue Qian, Liyang Xie, Dayan Huang, Xiaowei Yin, Systems Reliability Analysis and Fault Diagnosis Based on Bayesian Networks, 2009 International Work-shop on Intelligent Systems and Applications, 23-24 May (2009) 1 - 4.

[7] Qian Wenxue, Yin Xiaowei, Xie Liyang, System Reliability Allocation Based on Bayesian Network, Applied Mathematics \& Information Sciences, 6 (2012) 681-687.

[8] Ciarlet, Phillippe G., the Finite Element Method for Elliptic Problems, Amsterdam: North-Holland, 1978.

[9] Qian Wenxue, Yin Xiaowei, Xie Liyang, Reliability Modeling and Assessment of Component with Multiple Weak Sites under Complex Loading, Mathematical Problems in Engineering, 2014, Article ID 583803, 9 pages. 